\title{
A Universal Noise Removal Algorithm with an Impulse Detector
}

\author{
Roman Garnett, Timothy Huegerich, Charles Chui, Fellow, IEEE, and Wenjie He*, Member, IEEE
}

\begin{abstract}
We introduce a local image statistic for identifying noise pixels in images corrupted with impulse noise of random values. The statistical values quantify how different in intensity the particular pixels are from their most similar neighbors. We continue to demonstrate how this statistic may be incorporated into a filter designed to remove additive Gaussian noise. The result is a new filter capable of reducing both Gaussian and impulse noises from noisy images effectively, which performs remarkably well, both in terms of quantitative measures of signal restoration and qualitative judgements of image quality. Our approach is extended to automatically remove any mix of Gaussian and impulse noise.
\end{abstract}

Index Terms - impulse noise, Gaussian noise, mixed noise, image restoration, nonlinear filters, bilateral filter, denoising

\section{INTRODUCTION}

$\mathbf{N}$ OISE can be systematically introduced into images during acquisition and transmission. A fundamental problem of image processing is to effectively remove noise from an image while keeping its features intact. The nature of the problem depends on the type of noise added to the image. Fortunately, two noise models can adequately represent most noise added to images: additive Gaussian noise and impulse noise.

Additive Gaussian noise is characterized by adding to each image pixel a value from a zero-mean Gaussian distribution. Such noise is usually introduced during image acquisition. The zero-mean property of the distribution allows such noise to be removed by locally averaging pixel values. Ideally, removing Gaussian noise would involve smoothing inside the distinct regions of an image without degrading the sharpness of their edges. Classical linear filters, such as the Gaussian filter, smooth noise efficiently but blur edges significantly. To solve this problem, nonlinear methods have to be used, most notably the anisotropic diffusion technique of Perona and Malik [1]. Another interesting method is the bilateral filter studied by Tomasi and Manducci [2] based on the original idea of Overton and Weymouth [3]. The essence of these methods is to use local measures of an image to quantitatively detect edges and to smooth them less than the rest of the image.

Impulse noise is characterized by replacing a portion of an image's pixel values with random values, leaving the remainder unchanged. Such noise can be introduced due to transmission errors. The most noticeable and least acceptable pixels in the noisy image are then those whose intensities are much different from their neighbors.

The Gaussian noise removal methods mentioned above cannot adequately remove such noise because they interpret the noise

This work was supported by the National Science Foundation under grants CCR-0098331 and CCR-9988289 and by the Army Research Office under grant DAAD 19-00-1-0512.

R. Garnett is with the Departments of Mathematics and Computer Science, Washington University in Saint Louis, Saint Louis, MO 63130. Email: roman@math.wustl.edu.

T. Huegerich is with the Department of Physics, Rice University, Houston, TX 77005. Email: hugetim@alumni.rice.edu.

C. Chui and W. He are with the Department of Mathematics and Computer Science, University of Missouri-Saint Louis, Saint Louis, MO 63121. C. Chui is also with the Department of Statistics, Stanford University, Stanford, CA, 94305. Email: ckchui@stanford.edu, he@cs.umsl.edu. pixels as edges to be preserved. For this reason, a separate class of nonlinear filters have been developed specifically for the removal of impulse noise; many are extensions of the median filter [4], [5], or otherwise use rank statistics [6], [7], [8]. The common idea among these filters is to detect the impulse pixels and replace them with estimated values, while leaving the remaining pixels unchanged. When applied to images corrupted with Gaussian noise, however, such filters are not effective, and in practice leave grainy, visually disappointing results.

Not much work has been carried out on building filters that can effectively remove both Gaussian and impulse noise, or any mixture thereof. Such "mixed noise" could occur, for instance, when sending an already noisy image over faulty communication lines. Peng and Lucke suggested a fuzzy filter designed specifically for mixed noise [9]. Additionally, in 1996 Abreu, et al., proposed the medianbased SD-ROM filter to remove impulse noise, and their method proved quite effective [10]. They also gave quantitative measures demonstrating the SD-ROM filter's ability to remove Gaussian noise as well as mixed Gaussian and impulse noise. Although their filter has impressive quantitative results, when applied to images with Gaussian or mixed noise it often produces visuallydisappointing output similar to that of other median-based filters.

In this paper we introduce a framework for creating a universal noise removal filter that is based on a simple statistic to detect impulse noise pixels in an image. Instead of applying the "detect and replace" methodology of most impulse noise removal techniques, we show how to integrate such a statistic into a filter designed to remove Gaussian noise. The behavior of the filter can be adaptively changed to remove impulses while retaining the ability to smooth Gaussian noise. Additionally, the filter can be easily adapted to remove mixed noise.

This paper is arranged as follows: in the next section we introduce a local image statistic for detecting impulses. In section III we briefly explain the bilateral filter and describe how to incorporate the statistic into the filter to create a universal filter. Finally, in section IV we provide visual examples and numerical results that demonstrate our method's soundness.

\section{A New Statistic FOR Detecting IMPUlses}

\section{A. Noise models}

Throughout this paper, we will use standard matrix notation for images. For example, when $u$ is an image, $u_{i, j}$ will represent the intensity value of $u$ at the pixel location $(i, j)$ in the image domain. For the case of additive Gaussian noise, the noisy image, $u$, is related to the original image, $u^{0}$, by

$$
u_{i, j}=u_{i, j}^{0}+n_{i, j}
$$

where each noise value $n$ is drawn from a zero-mean Gaussian distribution.

Impulse noise, again to be denoted by $n$, is characterized by replacing a portion of the original pixel values of the image with intensity values drawn from some distribution, usually either a uniform or discrete distribution $n$ over the intensity range. 
Throughout this paper we consider the uniform noise distribution model, although the methods we discuss could be used without modification for the discrete model. ${ }^{1}$ Therefore, for images corrupted with impulse noise, the noisy image $u$ is related to the original image $u^{0}$ by

$$
u_{i, j}= \begin{cases}n_{i, j} & \text { with probability } p \\ u_{i, j}^{0} & \text { with probability }(1-p)\end{cases}
$$

\section{B. Motivation of the impulse detection scheme}

The problem of deciding which pixels in an image are impulses is clearly not well-defined. In particular, Roy Lichtenstein or George Seurat might be dismayed if he saw one of his paintings "denoised." Therefore we must be content with detecting pixels that are like impulses, that is, pixels that vary greatly in intensity from most surrounding pixels. Thankfully, uncorrupted natural images rarely contain details isolated to a single pixel and generally have few impulse-like pixels.

Impulse noise removal methods use many different techniques to determine whether a given pixel is an impulse in this sense. These approaches vary in complexity from being relatively simple to highly complex. The most basic impulse detectors are based on two-state methods that attempt to definitively characterize each image pixel as either an impulse or an unaffected pixel. The underlying goal of these two-state methods is to find pixels that are significant outliers when compared to their neighbors. One of the simplest and most intuitive method is to compare a pixel's intensity with the median intensity in its neighborhood, as in [5]. Other methods, such as the two-state SD-ROM filter of Abreu, et. al [10] and the recent CSAM filter of Pok, et. al [8], use more complex criteria to judge whether a pixel is an impulse. The advantage of these two-state methods is their simplicity, which makes them easily customizable.

More complex methods are naturally more successful for detecting impulses in general, but there is a tradeoff for this improvement in detection. The most complicated methods require training procedures to make an optimal classification based on measures of pixels and their neighbors. Methods that require training are bound to be less easily controlled and more unpredictable than simpler methods. If a method is trained on an image with many impulse-like pixels (such as an image with many fine details), its ability to detect and remove actual impulse noise from a different image will be inhibited. On the other hand, if the method is trained on a smooth image with few impulse-like pixels, it will overly smooth an image with many fine details that could otherwise be preserved. Even if care is taken to select an appropriate training image-assuming one is available - the performance of an automatically trained filter is essentially unpredictable.

An additional concern with existing methods arises from the fact that when impulse noise is introduced to an image, a portion of the pixels will be replaced with intensities only slightly different from their original values. Two-state detectors, with or without training, will most likely fail to detect such small impulses since they look exclusively for large outliers. They remove the most conspicuous noise, but the lesser impulses remain, creating a grainy appearance. In response to this problem, we adopt a continuous function to represent how impulse-like a particular pixel may be.

\footnotetext{
${ }^{1}$ In contrast, Conditional Signal-Adaptive Median (CSAM) filtering [8], is designed for salt-and-pepper noise, a discrete impulse noise model in which the noisy pixels take only the values 0 and 255 . It can remove salt-and-pepper type noise pixels very well, but it cannot perform similarly for uniformly distributed impulse noise and is outperformed by even the median filter.
}

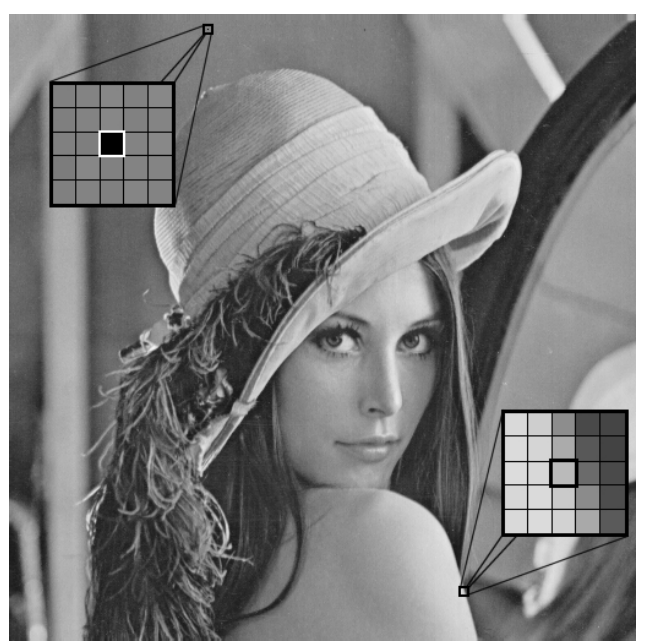

Fig. 1. Closeups of an artificially added impulse (upper left), and a typical edge pixel (lower right). ROAD of impulse: 525; ROAD of edge pixel: 88.

$$
\left(\begin{array}{rrr}
213 & 171 & 88 \\
216 & 186 & 107 \\
218 & 202 & 139
\end{array}\right) \rightarrow \quad\left(\begin{array}{ccc}
\mathbf{2 7} & \mathbf{1 5} & 98 \\
\mathbf{3 0} & - & 79 \\
32 & \mathbf{1 6} & 47
\end{array}\right)
$$

Original Neighborhood $\swarrow$ Absolute Differences

$$
\begin{gathered}
r_{1}=15, r_{2}=16 \\
r_{3}=27, r_{4}=30
\end{gathered} \quad \rightarrow \quad \begin{gathered}
\mathrm{ROAD}=\sum_{i=1}^{4} r_{i} \\
15+16+27+30=88
\end{gathered}
$$

The four smallest absolute differences
Final Calculation of ROAD
Fig. 2. Demonstrating how to calculate ROAD.

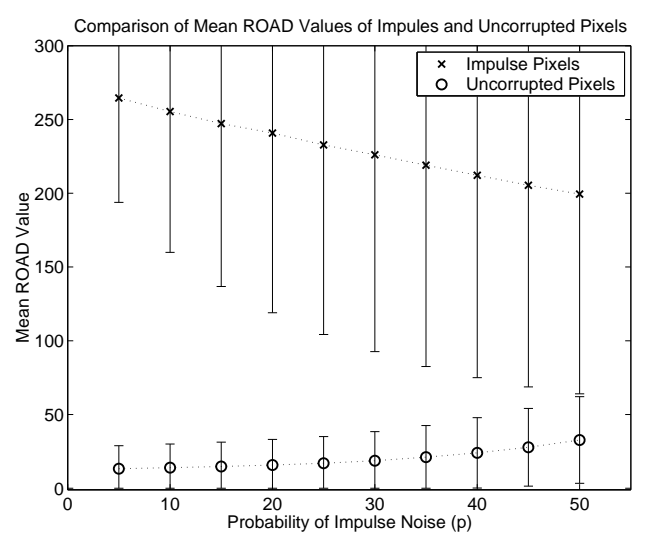

Fig. 3. The mean ROAD values of noise pixels and uncorrupted pixels in the Lena image as a function of the impulse noise probability, with standard deviation error bars demonstrating the significance of the difference.

By considering the magnitude of this function, we can adapt the behavior of the filter in a straightforward way according to how impulse-like each pixel is.

\section{Definition of the ROAD statistic}

Let $\mathbf{x}=\left(x_{1}, x_{2}\right)$ be the location of the pixel under consideration, and let

$$
\Omega_{\mathbf{x}}(\mathrm{N}):=\{\mathbf{x}+(i, j):-N \leq i, j \leq N\}
$$


be the set of points in a $(2 \mathrm{~N}+1) \times(2 \mathrm{~N}+1)$ neighborhood centered at $\mathbf{x}$ for some positive integer $N$. In the following discussion, let us only consider $N=1$, though the same procedure can be extended to any $N>1$. Hence,

$$
\Omega_{\mathbf{x}}^{0}=\Omega_{\mathbf{x}}(1) \backslash\{\mathbf{x}\},
$$

represents the set of points in a $3 \times 3$ deleted neighborhood of $\mathbf{x}$. For each point $\mathbf{y} \in \Omega_{\mathbf{x}}^{0}$, define $d_{\mathbf{x}, \mathbf{y}}$ as the absolute difference in intensity of the pixels between $\mathbf{x}$ and $\mathbf{y}$, i.e.

$$
d_{\mathbf{x}, \mathbf{y}}=\left|u_{\mathbf{x}}-u_{\mathbf{y}}\right| .
$$

Finally, sort the $d_{\mathbf{x}, \mathbf{y}}$ values in increasing order and define

$$
\operatorname{ROAD}_{\mathrm{m}}(\mathbf{x})=\sum_{i=1}^{m} r_{i}(\mathbf{x}),
$$

where $2 \leq m \leq 7$ and

$$
r_{i}(\mathbf{x})=i^{t h} \text { smallest } d_{\mathbf{x}, \mathbf{y}} \text { for } \mathbf{y} \in \Omega_{\mathbf{x}}^{0} .
$$

We call the statistic defined in (2) ROAD ("Rank-ordered Absolute Differences"). In this paper, we will consider $m=4$ only, and set $\operatorname{ROAD}(\mathbf{x})=\operatorname{ROAD}_{4}(\mathbf{x})$.

The ROAD statistic provides a measure of how close a pixel value is to its four most similar neighbors. The logic underlying the statistic is that unwanted impulses will vary greatly in intensity from most or all of their neighboring pixels, whereas most pixels composing the actual image should have at least half of their neighboring pixels of similar intensity, even pixels on an edge. Fig. 1 shows examples from the Lena image comparing a typical impulse noise pixel to an edge pixel. Notice that the edge pixel has neighbors of similar intensity despite forming part of an edge, and thus has a significantly lower ROAD value. Fig. 2 demonstrates how the latter value was calculated.

We illustrate numerically that the ROAD statistic is a good indicator of impulse noise. Ideally we want our statistic to be very high for impulse noise pixels and much lower for uncorrupted pixels. Fig. 3 displays quantitative results from the Lena image. The upper dashed line represents the mean ROAD value for noise pixels as a function of the amount of impulse noise added, and the lower dashed line represents the mean ROAD value for uncorrupted pixels. The noise pixels consistently have much higher mean ROAD values than the uncorrupted pixels, whose mean ROAD values remain nearly constant even with very large amounts of noise.

\section{INTRODUCING THE ROAD AND TRILATERAL FILTERS}

It would be relatively simple to introduce the ROAD statistic into many existing filtering techniques, allowing them to detect and properly handle impulse-like pixels in a noisy image. For example, one could modify the popular anisotropic diffusion method to utilize the ROAD statistic. Below we describe how one might extend the bilateral filter to create a filter capable of removing both impulse and additive Gaussian noise from images. We begin with a brief introduction to the bilateral filter.

\section{A. The bilateral filter}

The bilateral filter, as described in [2], applies a nonlinear filter to $u$ to remove Gaussian noise while retaining the sharpness of edges. Each pixel is replaced by a weighted average of the intensities in a $(2 \mathrm{~N}+1) \times(2 \mathrm{~N}+1)$ neighborhood. The weighting function is designed to smooth in regions of similar intensity while keeping edges intact, by heavily weighting those pixels that are both near the central pixel spatially and similar to the central pixel radiometrically.
More precisely, let $\mathbf{x}$ be the location of the pixel under consideration, and let

$$
\Omega=\Omega_{\mathbf{x}}(N)
$$

be the pixels in a $(2 \mathrm{~N}+1) \times(2 \mathrm{~N}+1)$ neighborhood of $\mathbf{x}$. The weight of each $\mathbf{y} \in \Omega$ with respect to $\mathbf{x}$ is the product of two components, one spatial and one radiometric:

$$
w(\mathbf{x}, \mathbf{y})=w_{S}(\mathbf{x}, \mathbf{y}) w_{R}(\mathbf{x}, \mathbf{y})
$$

where

$$
w_{S}(\mathbf{x}, \mathbf{y})=e^{-\frac{|\mathbf{x}-\mathbf{y}|^{2}}{2 \sigma_{S}^{2}}}
$$

and

$$
w_{R}(\mathbf{x}, \mathbf{y})=e^{-\frac{\left|u_{\mathbf{x}}-u_{\mathbf{y}}\right|^{2}}{2 \sigma_{R}^{2}}}
$$

The weights must be normalized, so the restored pixel $\tilde{u}_{\mathbf{x}}$ is given by

$$
\tilde{u}_{\mathbf{x}}=\frac{\sum_{\mathbf{y} \in \Omega} w(\mathbf{x}, \mathbf{y}) u_{\mathbf{y}}}{\sum_{\mathbf{y} \in \Omega} w(\mathbf{x}, \mathbf{y})}
$$

The $w_{S}$ weighting function decreases as the spatial distance between $\mathbf{x}$ and $\mathbf{y}$ increases, and the $w_{R}$ weighting function decreases as the radiometric "distance" between the intensities $u_{\mathbf{x}}$ and $u_{\mathbf{y}}$ increases. The spatial component of the weight decreases the influence of pixels far away from $\mathbf{x}$ to generally reduce blurring, while the radiometric component diminishes the influence of pixels with significantly different intensities to keep the edges of distinct image regions sharp. Notice that the $w_{S}$ and $w_{R}$ weighting functions need not be Gaussians-any suitable nonnegative functions that decrease to zero may be used instead.

In our particular weighting functions, the parameters $\sigma_{S}$ and $\sigma_{R}$ control the behavior of the weights. They are the values at which the respective Gaussian weighting functions take their maximum derivatives, so they serve as rough thresholds for identifying pixels sufficiently close spatially or radiometrically. Note, in particular, that as $\sigma_{R} \rightarrow \infty$ and radiometric differences are rendered irrelevant by this high threshold, the bilateral filter approaches a Gaussian filter of standard deviation $\sigma_{S}$. As both $\sigma_{R}, \sigma_{S} \rightarrow \infty$ so that all neighboring pixels easily meet both thresholds, the bilateral filter approaches the mean filter.

The idea for bilateral filtering was originally introduced in 1979 by Overton and Weymouth [3] as a simple image preprocessing tool to remove noise. In 1998, Tomasi and Manduchi [2] provided the name "bilateral filtering" and improved the method in several aspects. Specifically, they used Gaussian functions in the weighting functions (instead of the rational functions used in [3]) to improve the filter's performance, analyzed the interaction between the weighting functions, and proposed metrics to be used for color images. Elad [11] established a connection between bilateral filtering and several other methods in terms of minimizing functionals.

\section{B. A new weighting function}

We incorporate the ROAD statistic into the bilateral filtering framework by introducing a third weighting function influenced by how impulse-like each pixel of the image is. The "impulsive" weight, $w_{I}$, at a point $\mathbf{x}$ is given by:

$$
w_{I}(\mathbf{x})=e^{-\frac{\operatorname{ROAD}(\mathbf{x})^{2}}{2 \sigma_{I}^{2}}} .
$$

The $\sigma_{I}$ parameter determines the approximate threshold above which to penalize high ROAD values. 

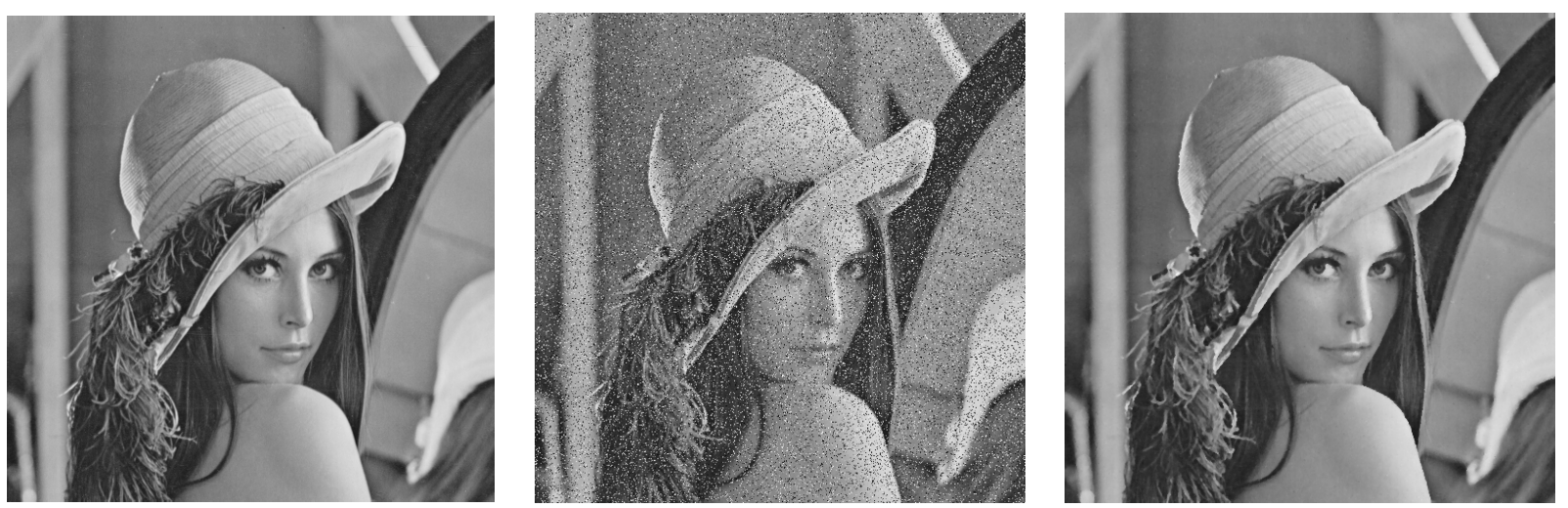

Fig. 4. The original Lena image, the image corrupted with impulse noise $(p=20 \%)$, and the result after trilateral filtering.

We would like to integrate this impulsive component into a nonlinear filter designed to weight pixels based on their spatial, radiometric, and impulsive properties. Unfortunately, the impulsive component is not directly compatible with the radiometric component already present in the bilateral filter. To illustrate this observation, let us consider black impulses on a white background. If the point $\mathrm{x}$ under consideration were such an impulse, the radiometric component would weight any other black impulses in the neighborhood of $\mathbf{x}$ much more than the white background pixels we desire. As a result, the black impulses remain black impulses. The radiometric weight works contrary to our goal because it was not designed to remove impulse noise. However, if used selectively, the radiometric weight can still be helpful for removing impulse noise. It can help smooth impulses that are only slightly different from their surrounding pixels without blurring edges, while the impulsive weight works to remove the larger outliers. If we can use the radiometric component for only small impulses, we can improve upon the common two-state methods for impulse noise removal by not only removing the larger outliers, but also smoothing away smaller impulses.

To add the impulsive weight while still retaining the radiometric component of the bilateral filter, we introduce a switch to determine how much to use the radiometric component in the presence of impulse noise. If $\mathbf{x}$ is the central pixel under consideration, and $\mathbf{y} \in \Omega_{\mathbf{x}}(N)$ is a pixel in the neighborhood of $\mathbf{x}$, we define the "joint impulsivity" $J$ of $\mathbf{y}$ with respect to $\mathbf{x}$ as

$$
J(\mathbf{x}, \mathbf{y})=1-e^{-\left(\frac{\operatorname{ROAD}(\mathbf{x})+\operatorname{ROAD}(\mathbf{y})}{2}\right)^{2} / 2 \sigma_{J}^{2}}
$$

The $J(\mathbf{x}, \mathbf{y})$ function assumes values in $[0,1]$. The $\sigma_{J}$ parameter controls the shape of the function. Again, any suitably nonnegative function that decreases to zero may be used in place of the Gaussian. If at least one of $\mathbf{x}$ or $\mathbf{y}$ is impulse-like and has a high ROAD value with respect to $\sigma_{J}$, then $J(\mathbf{x}, \mathbf{y}) \approx 1$. If neither pixel is impulse-like, and thus neither has a high ROAD value, then $J(\mathbf{x}, \mathbf{y}) \approx 0$.

We would like to use the radiometric weight more heavily when $J(\mathbf{x}, \mathbf{y}) \approx 0$ to smooth regions without large impulses and less heavily when $J(\mathbf{x}, \mathbf{y}) \approx 1$, because if either pixel is an impulse, the radiometric weight fails to function correctly as illustrated above. Conversely, we would like to use the impulsive weight less heavily when $J(\mathbf{x}, \mathbf{y}) \approx 0$ and more heavily when $J(\mathbf{x}, \mathbf{y}) \approx 1$, to suppress large impulses. With this in mind, we define the final, "trilateral" weight of $\mathbf{y}$ with respect to the central point $\mathbf{x}$ as:

$$
w(\mathbf{x}, \mathbf{y})=w_{S}(\mathbf{x}, \mathbf{y}) w_{R}(\mathbf{x}, \mathbf{y})^{1-J(\mathbf{x}, \mathbf{y})} w_{I}(\mathbf{y})^{J(\mathbf{x}, \mathbf{y})} .
$$

Referring to the Gaussian forms of $w_{R}$ and $w_{I}$, we see that raising these functions to the specified exponents has the effect of modifying their effective standard deviations or "thresholds". When $J(\mathbf{x}, \mathbf{y}) \approx 1$ so that $1-J(\mathbf{x}, \mathbf{y}) \approx 0$, the radiometric threshold becomes very large so that radiometric differences become irrelevant, while the impulsive weight is unaffected. When $J(\mathbf{x}, \mathbf{y}) \approx 0$, the opposite happens and only the radiometric weight is used to distinguish pixels because the effective impulsive threshold is so high. In this way, the appropriate weighting function is applied on a pixel-by-pixel basis. We will call the nonlinear filter of form (7) with the weighting function $w(\mathbf{x}, \mathbf{y})$ given in (10) the "trilateral filter," since it combines three different measures of neighboring pixels in determining its weights.

In general, the trilateral weighting function works well to remove impulse noise without compromising the bilateral filter's ability to remove Gaussian noise. For images with no impulse noiseand thus few points with high ROAD values-the $J(\mathbf{x}, \mathbf{y})$ term in (10) effectively "shuts off" the impulsive component of the weight and only uses the radiometric and spatial weights. Essentially, the trilateral filter reverts to the bilateral filter when processing images without impulse noise.

The $J(\mathbf{x}, \mathbf{y})$ function also allows for future work to further extend the filter. Specifically, we are exploring methods to automatically choose the control parameters $\sigma_{S}, \sigma_{R}, \sigma_{I}$, and $\sigma_{J}$ locally. This would allow us to remove different types of noise from the same image, as well as mixed Gaussian and impulse noise in a single pass.

We have found that even for very high levels of impulse noise, one pass of the trilateral filter will remove almost all of the noise. However for $p>25 \%$, a few spots of unremoved impulses often remain. This happens because impulses sometimes "clump" together in the original noisy image to form regions of similar intensity so large that they are mistaken for meaningful features. To remove such residual spots, it is often helpful to process the image with an iterative version of the filter. We simply run the image through the trilateral filter several times, using the output of the previous iteration as the input of the next. For low and moderate levels of noise $(p \leq 25 \%)$, one iteration is sufficient and usually provides the best results. For high levels of noise $(p>25 \%)$, applying two to five iterations provides better results.

\section{Removing Mixed Gaussian and Impulse Noise}

The trilateral filter can be easily extended to remove any mixture of Gaussian and impulse noise. The ideal solution would be to locally vary parameters so that they are finely tuned to remove the 


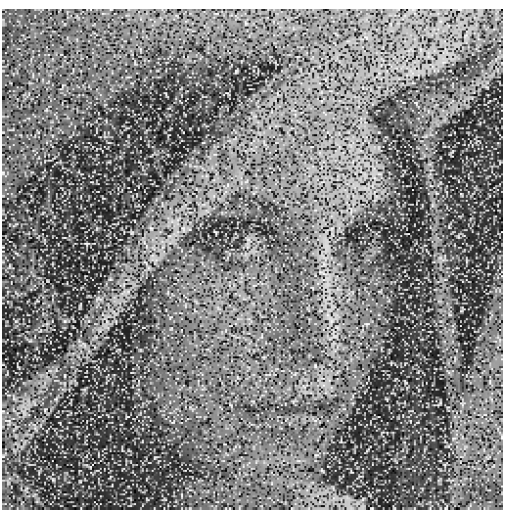

Closeup of Lena image corrupted with impulse noise $(p=50 \%)$

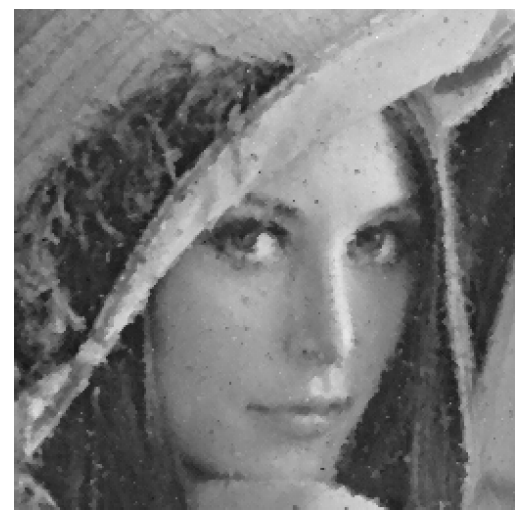

Restored with SD-ROM filter with training

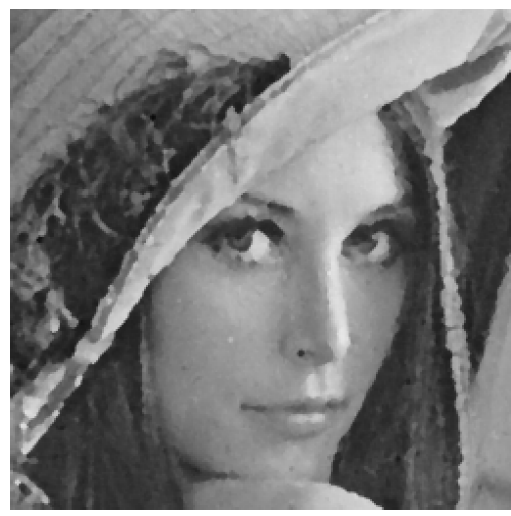

Restored with trilateral filter

Fig. 5. The Lena image corrupted with a high level of impulse noise and the results of applying several filters.

TABLE I

RESULTS AFTER APPLYING VARIOUS FILTERS TO IMAGES CORRUPTED WITH IMPULSE NOISE

\begin{tabular}{|c|c|c|c|c|c|c|c|c|}
\hline \multirow[b]{2}{*}{ Method } & \multicolumn{4}{|c|}{ Lena Image } & \multicolumn{4}{|c|}{ Bridge Image } \\
\hline & $p=20 \%$ & $p=30 \%$ & $p=40 \%$ & $p=50 \%$ & $p=20 \%$ & $p=30 \%$ & $p=40 \%$ & $p=50 \%$ \\
\hline $3 \times 3$ Median Filter & $31.23 \mathrm{~dB}$ & $28.05 \mathrm{~dB}$ & $24.61 \mathrm{~dB}$ & $21.53 \mathrm{~dB}$ & $24.76 \mathrm{~dB}$ & $23.14 \mathrm{~dB}$ & $21.20 \mathrm{~dB}$ & $19.04 \mathrm{~dB}$ \\
\hline $5 \times 5$ Median Filter & $29.87 \mathrm{~dB}$ & $28.97 \mathrm{~dB}$ & $27.61 \mathrm{~dB}$ & $25.45 \mathrm{~dB}$ & $23.26 \mathrm{~dB}$ & $22.73 \mathrm{~dB}$ & $21.95 \mathrm{~dB}$ & $20.72 \mathrm{~dB}$ \\
\hline $\begin{array}{l}\text { Median Filter with } \\
\text { Adaptive Length }{ }^{a}\end{array}$ & $\mathrm{~dB}$ & $29.49 \mathrm{~dB}$ & $\mathrm{~dB}$ & AB & 25 & $23.70 \mathrm{~dB}$ & $\mathrm{~dB}$ & $21.03 \mathrm{~dB}$ \\
\hline $\begin{array}{c}\text { Sun and Neuvo } \\
\text { Switching Scheme I }{ }^{a, b}[5]\end{array}$ & $32.15 \mathrm{~dB}$ & $29.69 \mathrm{~dB}$ & $27.97 \mathrm{~dB}$ & $25.54 \mathrm{~dB}$ & $26.16 \mathrm{~dB}$ & $24.34 \mathrm{~dB}$ & $22.75 \mathrm{~dB}$ & $21.02 \mathrm{~dB}$ \\
\hline $\begin{array}{c}\text { Rank Conditioned } \\
\text { Rank Selection Filter }{ }^{d}[7]\end{array}$ & $31.81 \mathrm{~dB}$ & $30.01 \mathrm{~dB}$ & $27.72 \mathrm{~dB}$ & $25.92 \mathrm{~dB}$ & $24.76 \mathrm{~dB}$ & $23.68 \mathrm{~dB}$ & $22.52 \mathrm{~dB}$ & $20.88 \mathrm{~dB}$ \\
\hline $\begin{array}{c}\text { SD-ROM } \\
\text { without training }{ }^{a, c}[10]\end{array}$ & $33.98 \mathrm{~dB}$ & $31.06 \mathrm{~dB}$ & $28.30 \mathrm{~dB}$ & $25.57 \mathrm{~dB}$ & $26.57 \mathrm{~dB}$ & $24.82 \mathrm{~dB}$ & $23.17 \mathrm{~dB}$ & $21.40 \mathrm{~dB}$ \\
\hline $\begin{array}{c}\text { SD-ROM } \\
\text { with training }{ }^{a, d}[10]\end{array}$ & $34.23 \mathrm{~dB}$ & $31.64 \mathrm{~dB}$ & $29.19 \mathrm{~dB}$ & $26.63 \mathrm{~dB}$ & $26.24 \mathrm{~dB}$ & $24.98 \mathrm{~dB}$ & $23.66 \mathrm{~dB}$ & $22.19 \mathrm{~dB}$ \\
\hline Trilateral Filter $^{e}$ & $35.03 \mathrm{~dB}$ & $33.16 \mathrm{~dB}$ & $31.36 \mathrm{~dB}$ & $29.44 \mathrm{~dB}$ & $27.55 \mathrm{~dB}$ & $25.99 \mathrm{~dB}$ & $24.55 \mathrm{~dB}$ & $23.17 \mathrm{~dB}$ \\
\hline
\end{tabular}

${ }^{a}$ Implemented recursively.

${ }^{b}$ Implemented using a $5 \times 5$ window for Lena with $p \geq 30 \%$, and using a $3 \times 3$ window otherwise

${ }^{c}$ Using the thresholds suggested in [10].

${ }^{d}$ The Lena image was trained on the "bridge" image with $35 \%$ noise, and the "bridge" image was trained on the Lena image with $35 \%$ noise.

${ }^{e}$ Implemented iteratively for $p \geq 30 \%$.

precise amount and type of noise present in each section of the image. This solution, however, would require a deep statistical study of the ROAD statistic for the automatic selection of parameters, and the best way to do this is not immediately clear.

A simpler, yet still quite effective solution to restore an image corrupted by mixed noise is to apply the trilateral filter twice with two different values of $\sigma_{S}$ - once with a smaller value of $\sigma_{S}$, to remove the impulse noise, and another time with a larger value of $\sigma_{S}$, to smooth the remaining Gaussian noise. A myriad of other options are available for altering the parameters between filtering, but our simple approach produces visually appealing results and only requires changing one parameter.

\section{Results}

We have extensively tested the noise removal capabilities of the trilateral filter and compared the results with several existing methods. Our method produced results superior to the other methods we tested in both visual image quality and quantitative measures of signal restoration. Among the many images we tested are the $512 \times 5128$ 8-bit grayscale Lena image, available online from Mike Wakin at Rice University [15], and the $512 \times 512$ "bridge" image, available online from the UEA Signal and Image Processing Group [16].

\section{A. Implementation and Testing Procedure}

Our implementation of the trilateral filter used a $5 \times 5$ window size and performed multiple iterations when it provided better results (for $p>25 \%$ ). Image boundaries were handled by assuming symmetric boundary conditions.

In each of our experiments we strove to be impartial while collecting data. For each method tested, we varied its parameters exhaustively (as suggested by its author(s)) to obtain the best possible result. Furthermore, to eliminate the bias created by different manifestations of noise, we created a standard set of noisy images. Five noisy images were created for each test image and noise level, and formed the common input to each method. The numerical results shown are the average results for these five images.

\section{B. Image Quality}

Our first goal was to ensure that our approach provides visually pleasing output. The trilateral filter can restore images corrupted with low to moderate levels of impulse noise $(p \leq 25 \%)$ with virtually unblemished results. Fig. 4 shows the Lena image corrupted with $20 \%$ impulse noise and the result after trilateral filtering. Comparing with the original, it is clear that the trilateral 


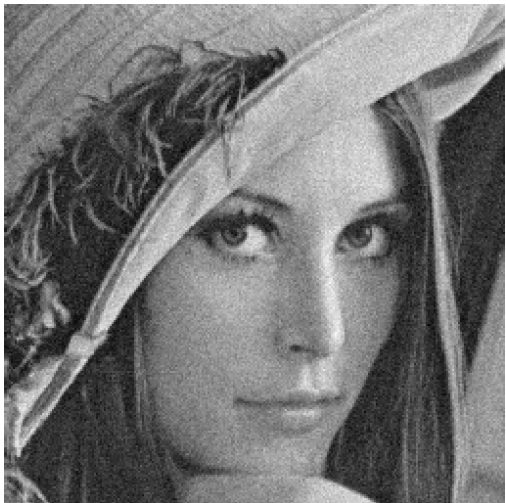

Image corrupted by Gaussian noise $(\sigma=10)$

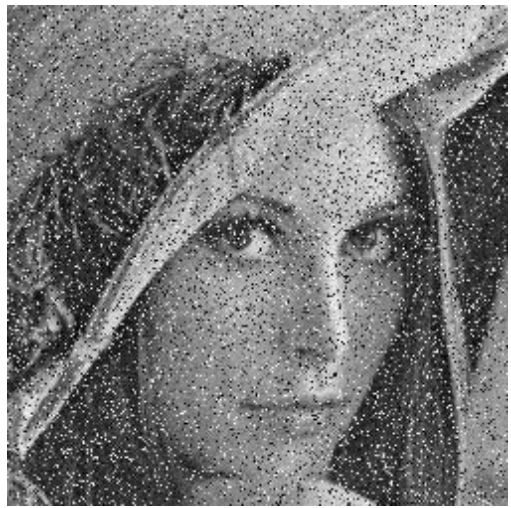

Image corrupted by mixed noise $(\sigma=10, p=20 \%)$

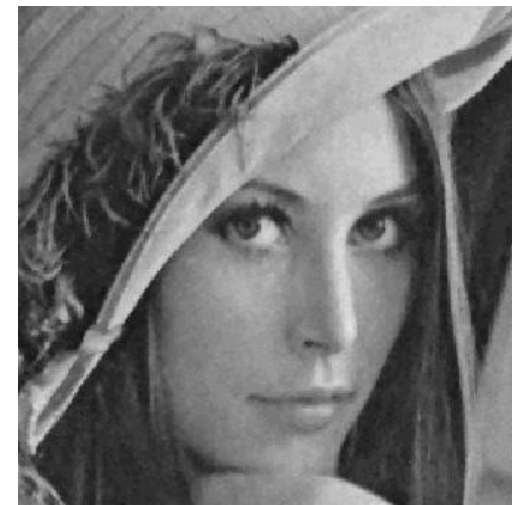

Restored with SD-ROM filter with training [10]

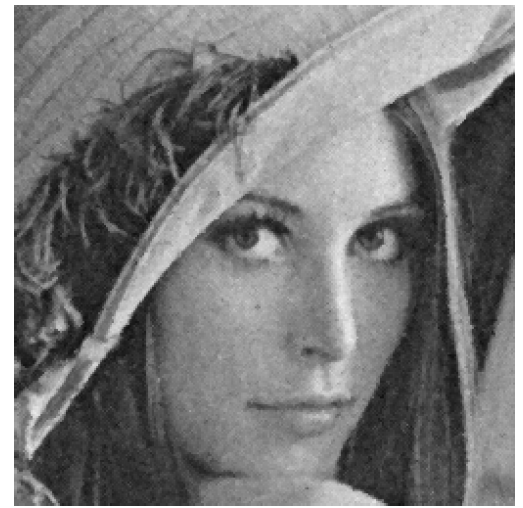

Restored with SD-ROM filter with training [10]

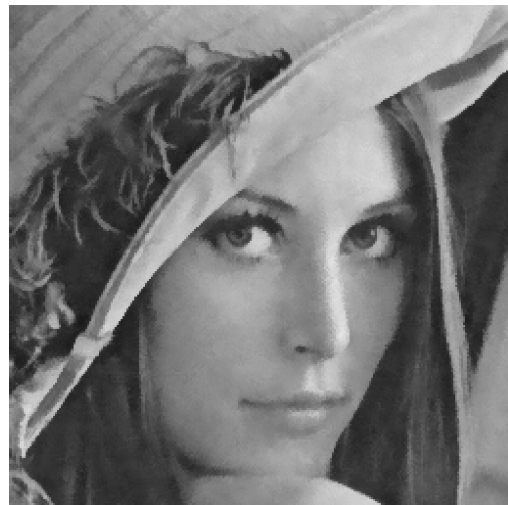

Restored with Trilateral filter

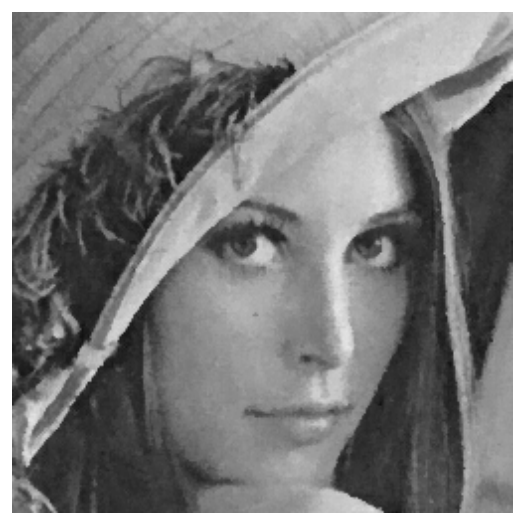

Restored with Trilateral filter

Fig. 6. Comparing the trilateral filter and SD-ROM filter on images with Gaussian noise and mixed Gaussian and impulse noise.

filter can eliminate a fair amount of noise while preserving edge boundaries and fine details.

Although exceptional for lower impulse noise levels, our filter truly excelled when treating highly corrupted $(p>25 \%)$ images. Fig. 5 shows the Lena image with 50\% impulse noise and compares the outputs of the trilateral filter with the output of the SD-ROM filter of Abreu, et al. The trilateral filter's output has fewer spots and other artifacts and is generally more pleasing.

We also verified that the trilateral filter retains the ability to remove Gaussian noise and that it can effectively remove mixed noise. Fig. 6 shows the Lena image corrupted with Gaussian noise and mixed noise and the outputs of the SD-ROM and trilateral filters. The trilateral filter continues to adequately suppress Gaussian noise, even after the introduction of the impulsive weight and the joint impulsivity function.

For both Gaussian and mixed noise, the trilateral filter leaves less noise in the restored image than the SD-ROM filter, and its output is generally more visually appealing. However, some fine details are lost in the process. For example, the individual strands of hair in the image are better kept in the SD-ROM filter's output, at the expense of a less smooth and less pleasing output.

\section{Signal Restoration}

Once the visual quality of images restored by the trilateral filter had been confirmed, we concentrated on directly comparable, quantitative measures of signal restoration. In particular, we measured the peak signal-to-noise ratio (PSNR). If $u^{0}$ is the original $m \times n$ image and $\tilde{u}$ is a restored image of $u^{0}$, the PSNR of $\tilde{u}$ is given by:

$$
\operatorname{PSNR}(\tilde{u})=10 \log _{10}\left(\frac{\sum_{i, j=1}^{m, n} 255^{2}}{\sum_{i, j=1}^{m, n}\left(\tilde{u}_{i, j}-u_{i, j}^{0}\right)^{2}}\right) .
$$

Larger PSNR values signify better signal restoration. We tested each method on impulse noise levels from $p=0 \%$ (that is, no noise) to $p=50 \%$ in steps of $5 \%$. Table I compares the mean PSNR values of the five restored test images for $p \in\{20 \%, 30 \%, 40 \%, 50 \%\}$. Again, the trilateral filter provided results with higher PSNR values than the results of the other methods tested, especially for very high levels of noise. In particular, for the Lena image with $50 \%$ noise, the trilateral filter produces PSNR values almost a full three decibels higher than the closest competing method.

We also compared the performance of the trilateral filter with the performance of the previously tested filters on images corrupted with Gaussian noise $(\sigma=10)$ and mixed noise $(\sigma=10, p=$ $20 \%$ ). The trilateral filter consistently yielded the highest PSNR for each image and noise level. Table II shows the results for the $3 \times 3$ median filter, the trilateral filter, and the previously-tested methods with training procedures: the RCRS and SD-ROM filters. Notice that the other methods generally performed only slightly better than the median filter for mixed noise.

\section{CONCLUSIONS}

Many noise removal algorithms, such as the bilateral filtering, tend to treat impulse noise as edge pixels, and hence end with 
TABLE II

PSNR VALUES (IN DECIBELS) OF NOISY IMAGES RESTORED WITH VARIOUS FILTERS

\begin{tabular}{|c|c|c||c|c|}
\hline \multirow{2}{*}{ Filter } & \multicolumn{2}{|c||}{ Gaussian Noise } & \multicolumn{2}{c|}{ Mixed Noise } \\
& \multicolumn{2}{|c|}{$\sigma=10$} & \multicolumn{2}{c|}{$p=20 \%, \sigma=10$} \\
\cline { 2 - 5 } & Lena & Bridge & Lena & Bridge \\
\hline $3 \times 3$ Median $^{\prime}$ & 30.92 & 25.93 & 29.02 & 24.20 \\
\hline RCRS $^{b}[7]$ & 31.00 & 27.38 & 29.55 & 24.46 \\
\hline SD-ROM $^{a, b}[10]$ & 32.20 & 28.85 & 29.87 & 25.10 \\
\hline \hline Trilateral $^{c}$ & 33.23 & 29.33 & 31.64 & 26.29 \\
\hline
\end{tabular}

${ }^{a}$ Implemented recursively.

${ }^{b}$ The Lena image was trained on the "bridge" image, and the "bridge" image was trained on the Lena image with the same level of noise.

${ }^{c}$ Implemented iteratively.

unsatisfactory results. In order to process impulse pixels and edge pixels differently, we introduce a new statistic based on rankordered absolute differences (ROAD) in some neighborhood of a pixel. This statistic represents how impulse-like a particular pixel is in the sense that the larger the impulse, the greater the ROAD value.

We then incorporate the ROAD statistic into the bilateral filtering by adding a third component to the weighting function. The new nonlinear filter is called the trilateral filter, whose weighting function contains spatial, radiometric, and impulsive components. The radiometric component combined with the spatial component smooths away Gaussian noise and smaller impulse noise; while the impulsive component removes larger impulses. A switch based on the ROAD statistic is adopted to adjust weight distribution between the radiometric and impulsive components. The resulting trilateral filter performs well in removing Gaussian and mixed noise as well as in removing impulse noise.

\section{REFERENCES}

[1] P. Perona and J. Malik, "Scale-space and edge detection using anisotropic diffusion," IEEE Trans. Pattern Anal. Machine Intell., vol. 12, pp. 629-639, 1990.

[2] C. Tomasi and R. Manduchi, "Bilateral filtering for gray and color images," Proc. 1998 IEEE Int. Conf. Computer Vision, pp. 839-846.

[3] K. J. Overton and T. E. Weymouth, "A noise reducing preprocessing algorithm," Proceedings of the IEEE Computer Science Conference on Pattern Recognition and Image Processing, pp. 498-507, Chicago, IL, 1979.

[4] H. Lin and A. N. Willson, Jr., "Median filters with adaptive length," IEEE Trans. Circuits Syst., vol. 35, pp. 675-690, June 1988.

[5] T. Sun and Y. Neuvo, "Detail-preserving median based filters in image processing," Patt. Recogn. Lett., vol. 15, pp. 341-347, Apr. 1994.

[6] A. C. Bovik, T. S. Huang, and D. C. Munson, "A generalization of median filtering using linear combinations of order statistics," IEEE Trans. ASSP, vol. 31, pp. 1342-1350, Dec. 1983.

[7] R. C. Hardie and K. E. Barner, "Rank conditioned rank selection filters for signal restoration," IEEE Trans. Image Processing, vol. 3, pp. 192206, Mar. 1994.

[8] G. Pok, J. Liu, and A. S. Nair, "Selective removal of impulse noise based on homogeneity level information," IEEE Trans. Image Processing, vol. 12, pp. 85-92, Jan. 2003.

[9] S. Peng and L. Lucke, "Multi-level adaptive fuzzy filter for mixed noise removal," Proc. IEEE Int. Symp. Circuits Syst., Seattle, WA, vol. 2, pp. 1524-1527, Apr. 1995

[10] E. Abreu, M. Lightstone, S. Mitra, and K. Arakawa, "A new efficient approach for the removal of impulse noise from highly corrupted images," IEEE Trans. Image Processing, vol. 5, pp. 1012-1025, June 1996.

[11] M. Elad, "On the origin of the bilateral filter and ways to improve it," IEEE Trans. Image Processing, vol. 11, pp. 1141-1151, Oct., 2002.

[12] R. L. Lagendijk, J. Biemond, and D. E. Boekee, "Regularized iterative image restoration with ringing reduction," IEEE Trans. Acoustics, Speech, Signal Processing, vol. 36, pp. 1874-1887, Dec. 1988.

[13] M. J. Black and G. Sapiro, "Edges as outliers: anisotropic smoothing using local image statistics," Scale-Space Theories in Comput. Vision, Second Int. Conf., Scale-Space '99, Corfu, Greece, LNCS 1682, pp. 259-270, Springer, Sept. 1999.

[14] J. Immerkær, "Fast Noise Variance Estimation," Comput. Vision and Image Understanding, vol. 64, pp. 300-302, Sept. 1996.

[15] M. Wakin, "Standard test images - Lena," available online: http: //www-ece.rice.edu/ wakin/images/.

[16] UEA Signal and Image Processing Group, "Standard Image Page," available online:

http://www.sys.uea.ac.uk/Research/researchareas/ imagevision/images_ftp/.

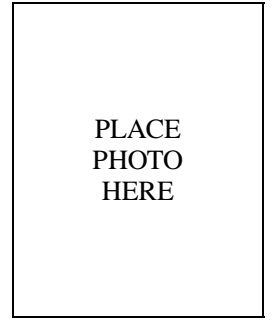

Roman Garnett recieved the A.B. degree in mathematics and the M.Sc. degree in computer science from Washington University in Saint Louis, May 2004.

The work for this paper was completed while he was a research aide at The Institute for Computational Harmonic Analysis at the University of Missouri-Saint Louis.

His research interests include image and video processing, and computer vision.

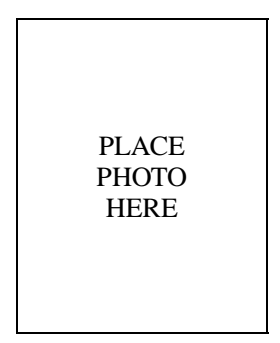

Timothy Huegerich recieved the B.S. degree in physics from Rice University in Houston in May 2004.

The work for this paper was completed while he was a research aide at The Institute for Computational Harmonic Analysis at the University of Missouri-Saint Louis.

His research interests include mathematical ecology and epidemiology.

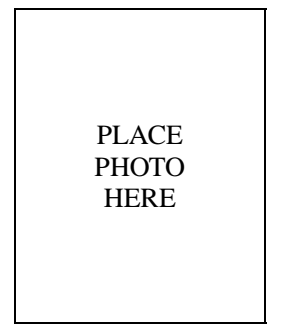

Charles Chui (Fellow, 94) received his higher education from the University of Wisconsin, Madison (B.S, M.S. and Ph.D.) and is currently $\mathrm{Cu}-$ rators' Professor at the University of Missouri, St. Louis, and Consulting Professor of Statistics at Stanford University. He is co-editor-in-chief of Applied and Computational Harmonic Analysis, and serves on the editorial board of seven other journals. In addition, he is editor of three book series, as well as editorial advisor of Elsevier book publication in Electrical Engineering. His research interests are in the areas of Approximation Theory, Computational Harmonic Analysis, Surface Subdivisions, and Mathematics of Imaging.

PLACE

PHOTO

HERE
Wenjie He received the B.S. degree in mathematics from Peking University, Beijing, China in 1988 and the Ph.D. degree in mathematics from the University of Georgia, Athens, Georgia in 1998.

$\mathrm{He}$ is currently Assistant Professor of Computer Science at the University of Missouri-Saint Louis. His research interests include wavelets, wavelet tight frames, computer graphics, and image processing. 